\title{
Face Detection Study based on Skin Color and Improved Adaboost Algorithm
}

\author{
Fan Chen $^{1}$, Jianxin Song ${ }^{2}$ \\ ${ }^{1}$ College of Telecommunication \& Information Engineering, Nanjing University of Posts \& \\ Telecommunications, Nanjing 210003, China \\ ${ }^{2}$ College of Telecommunication \& Information Engineering, Nanjing University of Posts \& \\ Telecommunications, Nanjing 210003, China
}

Keywords: skin color, AdaBoost, face detection, error rate, Haar

\begin{abstract}
For color images in a complex background, we cannot be able to detect faces quickly. So we put forward an algorithm, which is based on skin color feature and the improved AdaBoost algorithm. First, through the skin color detection to excluding large amounts of complex background of non-face, after that define the face candidate regions. Besides, when the image is darkness, we will increase the light treatment, afterwards use AdaBoost algorithm to detect the human face, to improve the accurate rate of face detection system and reduce the error rate. In addition, based on the AdaBoost algorithm of former research ,we add new Haar features and modify the weight of its update method, so under the condition of the less weak classifier, the AdaBoost algorithm's training speed much faster, and to prevent the excessive distribution in the process of the training. The experimental results show the proposed method has great improvement for face detection.
\end{abstract}

\section{Introduction}

In recent years, along with the rapid development of computers' speed, which makes the image processing technology has been widely applied in many fields, including intelligent monitoring, security trading, human-computer interaction and so on. Face detection [1-2] is an significant branch in pattern recognition, its means to find out all faces in the picture. In [3], according to a priori knowledge that the human face can be similar to an ellipse, using the generalized Hough transform to extract the elliptical shape information, then we can achieve the face detection. In [4], it established the face Mosaic model and relatively complete knowledge base, using the knowledge base put forward the fast face detection method. These methods can better cope with the variety environmental, and has a higher precision. But this kind of method usually needs to be done with the huge sample training and learning, how to complete high-speed and effective training, reduce the great amount of calculation, and increase the efficiency of the face detection, which are the urgent demand problem. In [5], put forward an Adaboost face detection algorithm based on the skin color features, In [6], combining with skin color detection and the improved Adaboost algorithm. Though, these methods have fast detection speed, its testing environment needs higher requirements, such as the simple background, good lighting, and so on, the imperfection of the face feature extraction is another weakness, which could not finish the high detection precision.

According to the above problem, this paper combines with the knowledge-based methods and the statistical analysis method. The first step is the pretreatment of the light, and color, put forward new Haar features and improved Adaboost algorithm. Through pretreatment of color and image enhancement, we can reduce the amount of calculations of face detection, new Haar features and the improved Adaboost algorithm can reduce the training time, and increase the accuracy of face detection. So it can overcome the defect of above methods, and complete higher efficiency and higher precision face detection. In this paper, the structure is as follows. Section 2 introduces the Adaboost algorithm. Section 3 introduces the improved Adaboost algorithm. Section 4 is the experiment, which verify the high speed of skin color processing and the efficiency and accuracy of the improved algorithm. Section 5 is a conclusion. 


\section{The algorithm of face detection}

\subsection{AdaBoost algorithm}

AdaBoost algorithm is a boosting algorithm, which has the ability of applicability. The main idea is that according to the same training set, we should train different weak classifier, then form a stronger final classifier (strong classifier) by putting these weak classifier together. The algorithm is achieved by changing the data distribution, and the weights of each sample are determined by the accuracy of training set of each sample and the overall classification of last time. Then training the classifier whose weight had been modified, and combining the trained classifiers, finally we will get the decision classifier.

Adaboost algorithm's steps are as follows, Set $n$ training samples are $\left\{\left(x_{1}, y_{1}\right),\left(x_{2}, y_{2}\right), \cdots,\left(x_{n}, y_{n}\right)\right\}, x_{i}$ is input of the training samples, $y_{i} \in\{0,1\}$ respectively represent positive samples and negative samples. Among them, $l$ is the number of positive samples and $m$ is the number of negative samples. The specific process are as follows,

(1)Initialize the weights of every sample, (2)For each $t=1, \cdots, T$ ( $T$ represents numbers of weak classifier),

(1) Normalize the weight, making them become a probability distribution.

$$
w_{t, i}=\frac{w_{t, i}}{\sum_{j=1}^{n} w_{t, j}}
$$

(2)For each feature $f$, training a weak classifier $h_{j}$ to calculate the weighted error rate of all corresponding characteristics of the weak classifier.

$$
\varepsilon_{j}=\sum_{i=1}^{n} w_{t}\left(x_{i}\right)\left|h_{j}\left(x_{i}\right) \neq y_{i}\right|
$$

(3) Select the best weak classifier $h_{t}$ (with minimum error rate)

(4)According to the optimal weak classifier, adjusting the weights

$$
w_{t+1, i}=w_{t, i} \beta_{t}^{1-\varepsilon_{i}}
$$

Among them, $\varepsilon_{i}=0$ represents classification correctly, $\varepsilon_{i}=1$ represents wrong.

(3) The final strong classifier

$$
\begin{aligned}
& h(x)=\left\{\begin{array}{lc}
1 & \sum_{t=1}^{T} \alpha_{t} h_{t}(x) \geq \frac{1}{2} \sum_{t=1}^{T} \alpha_{t} \\
0 & \text { otherwise }
\end{array}\right. \\
& \alpha_{t}=\log \frac{1}{\beta_{t}}
\end{aligned}
$$

\subsection{Advantage and disadvantage of AdaBoost}

AdaBoost face detection is a method based on integral image, cascaded detectors and AdaBoost algorithm [7]. AdaBoost don't need to know all the lower limit of the error probability of weak learning machine, but according to the classification situation of the current weak machine learning, to produce a right value. When using AdaBoost technology for learning, training error rate is a role of monotonic decline, in this way, as long as the basic properties of weak learning machine can be better than immediately speculation, and cycle enough time, then the error rate of final classifier can also be lower than any value, the conclusion has been proved in [8]. Experimental results show the efficiency of AdaBoost similar to the traditional Boost technology, but there is not any requirement for the lower limit of the error classification of weak learning machine, which can improve its usefulness.

Although Aadboost's detection rate is high, the training time of the whole system is surprised. 
According to [9], the system will spent weeks on training, and the iteration update weights coefficient of original Adaboost algorithm is fixed, for the different categories of samples, we can not process differently, which will give some training difficult samples high weight, and make the distribution of sample weight distortion and excessive allocation, finally lead to decrease the detectability of algorithm. Therefore, it is necessary to improve the original training algorithm, accelerate training time of the classifier and remove the phenomenon of excessive distribution of some samples.

\section{Improved Haar features and Adaboost algorithm}

\subsection{Haar features}

Viola, etc. put forward a kind of framework based on Boosted cascade of simple features to detect objects[10]. In 2002, Lienhart and Maydt [11] extended the Haar features. Figure 1 shows the commonly Haar features, its eigenvalue is the sum of black pixel gray value minus the sum of white pixel gray value. Haar features are mainly divided into edge feature, line features, point features and diagonal features.

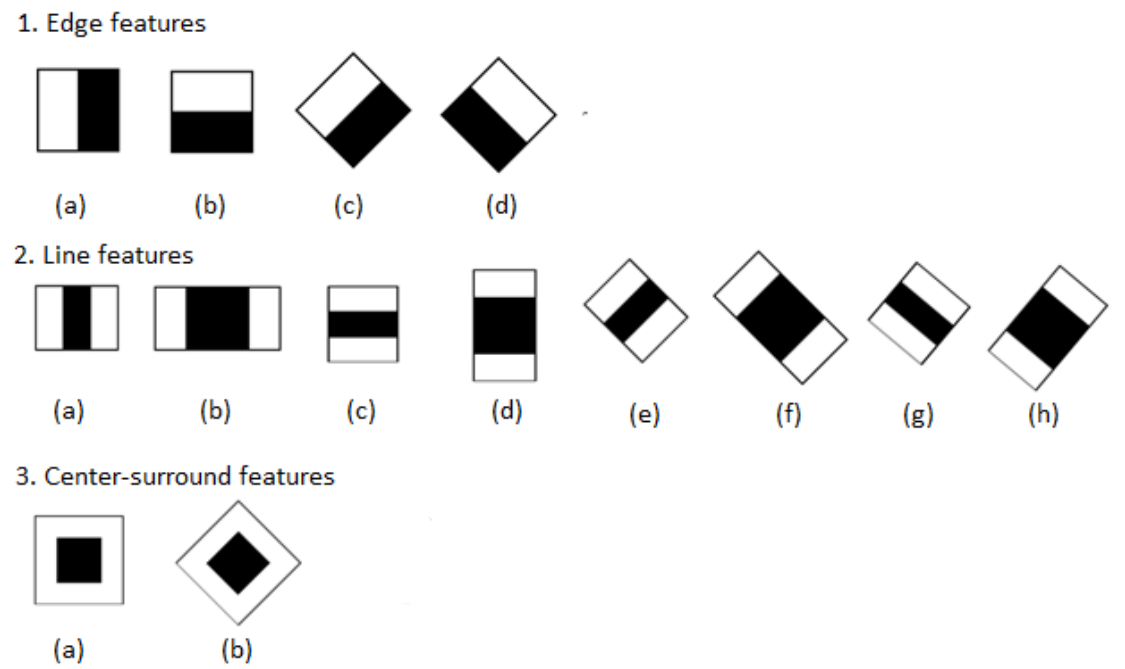

Figure 1 Haar features

\subsection{Haar features' improvement}

Due to the particularity of distribution of facial features, human eyes, nose and forehead become the most important part in face, however, the gray value of human eye and nose is low, and the grey value of forehead is high. According to the feature of existing Haar features, this paper puts forward two kinds of new Haar features, as is shown in figure 2.
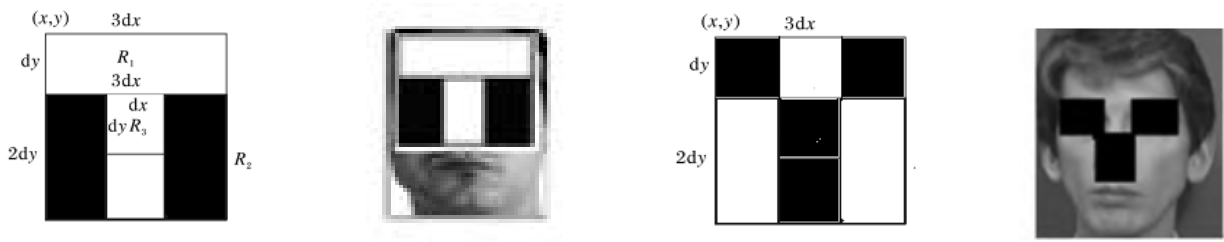

Figure 2 New Haar features

\subsection{Improved Adaboost algorithm}

According to original Adaboost algorithm, after each round of training, the weight of the sample will be changed, the training samples of failure will be given greater weight, then make the algorithm focused on samples of incorrect classification [12], when there are some samples that are difficult to practice, the algorithm will assign these difficulties' sample a high weight, which makes the sample weight distortion and excessive distribution. Because of the increasing times of training, it may undermine or missing the accurate classification rules, resulting decline in the algorithm's ability. In order to avoid the excessive distribution phenomenon in the process of training, this 
article adopts the grow new weight updating method: control the growth of right identify samples and wrong identify samples, set a threshold $H W_{\mathrm{t}}$ on each training, update the weights according to the sample is classified right or not and whether the threshold value is greater than the current weight $H W_{\mathrm{t}}$.

$$
w_{t+1, j}=\left\{\begin{array}{lll}
\frac{w_{t, j} \beta_{i}}{Z_{t}} & \text { if } & h_{i}\left(x_{i}\right)=y_{i} \\
\frac{w_{t, j} \beta^{-1}}{Z_{t}} & \text { if } & h_{i}\left(x_{i}\right) \neq y_{i}, w_{t, j} \leq H W_{t} \\
\frac{w_{t, j}}{Z_{t}} & \text { if } & h_{i}\left(x_{i}\right) \neq y_{i}, w_{t, j}>H W_{t}
\end{array}\right.
$$

$\beta_{t}=\frac{\varepsilon_{t}}{1-\varepsilon_{t}}, \quad Z_{t}$ is unit factor, which make $\sum_{j=1}^{n} w_{t, j}=1, H W_{t}$ is the new threshold of $t$ times training.

From the above algorithm's description, only when a sample is classified wrong, and the current value $W_{t}$ is less than the new threshold $H W_{t}$, the sample's weight will be increased, otherwise, its weight will be reduced. In this way, even if the difficult samples was classified wrong, the weight will not be increased too much, which can avoid the phenomenon of excessive distribution to a certain extent.

\section{Experiments}

In this paper, we used MIT face repository to train samples, true samples' number is 1,439 and false samples' is 4,381. Extracting 300 samples for the classifiers to detect samples. Test environment is dual core $2.2 \mathrm{GHz}$ CPU, $4 \mathrm{~GB}$ of memory, Windows 7 operating system, the platform is VS2010 with Opencv programming, part of the test results are shown in the figures.

Figure 3 and figure 4 don't use skin color detection, figure 5 and figure 6 use skin color detection. Table 1 is the comparison of the detection rate, table 2 is the detection rate and false detection rate.

The experimental results show that skin color detection can not only reduce the detection error rate but also speed up the detection, comparing this paper's algorithm with the original algorithm, the detection rate and error detection rate had improved.

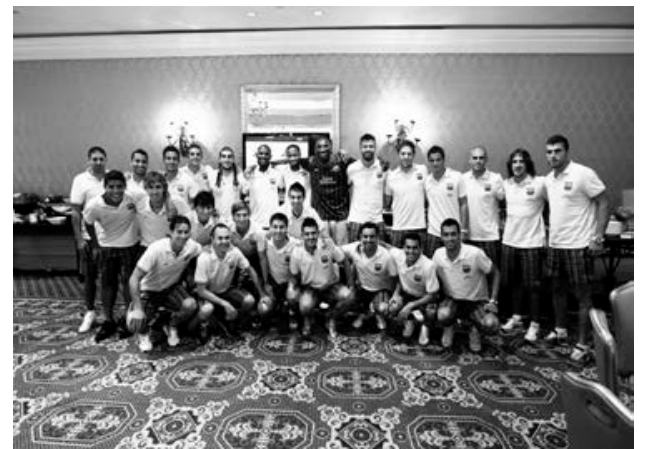

Figure 3 without skin color detection

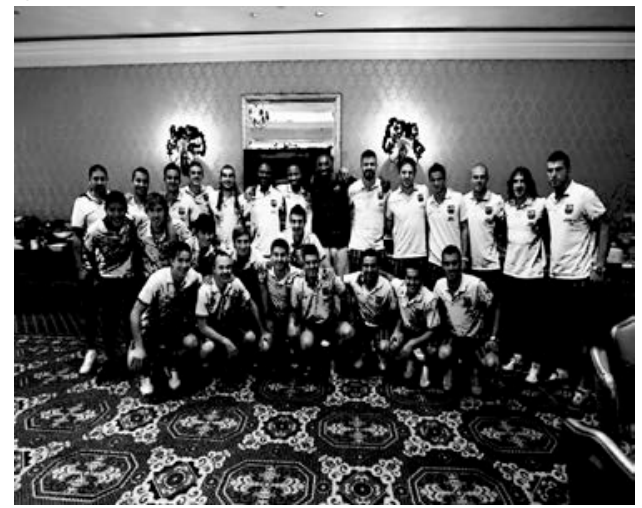

Figure 5 with skin color detection

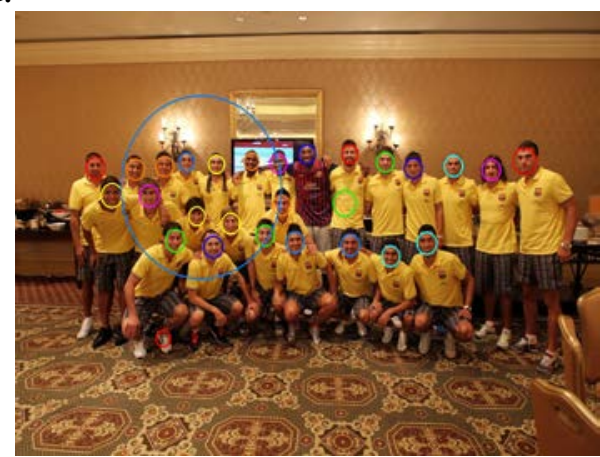

Figure 4 without skin color detection’s result

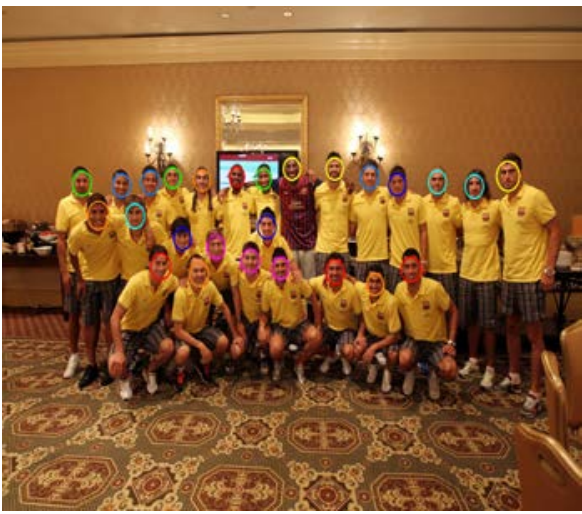

Figure 6 with skin color detection's result 


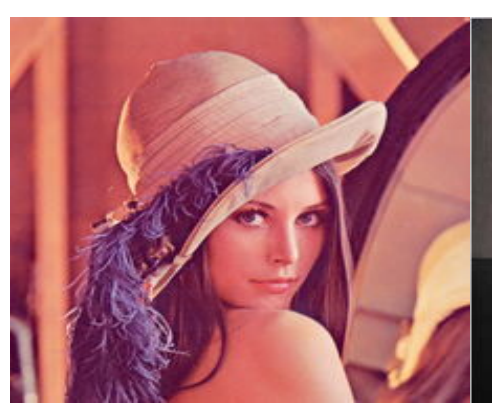

Figure a

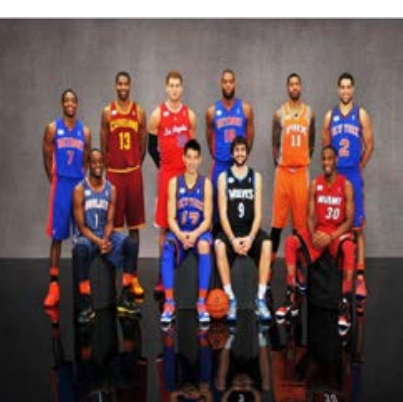

Figure b

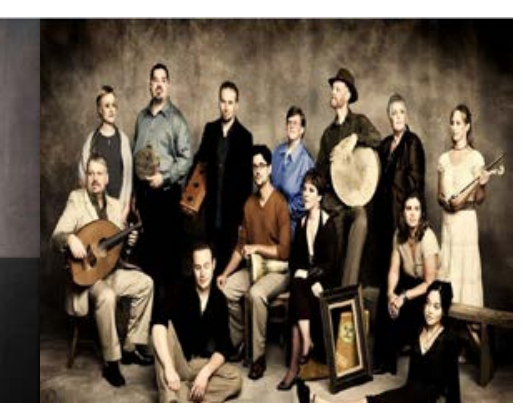

Figure c

Table 1 detection speed (ms)

\begin{tabular}{c|c|c|c}
\hline detection method & Figure a & Figure b & Figure c \\
\hline Adaboost & 113.547 & 1768.92 & 1785.60 \\
\hline Skin color+Adaboost & 103.186 & 1691.94 & 1435.83 \\
\hline
\end{tabular}

Table 2 detection rate

\begin{tabular}{c|c|c|c|c}
\hline detecting algorithm & samples & $\begin{array}{c}\text { detection } \\
\text { numbers }\end{array}$ & $\begin{array}{c}\text { detection } \\
\text { rate }\end{array}$ & $\begin{array}{c}\text { error } \\
\text { detection rate }\end{array}$ \\
\hline Adaboost & 300 & 260 & $86.6 \%$ & $9.46 \%$ \\
\hline Ipmrocvment+Adaboost & 300 & 284 & $94.6 \%$ & $4.38 \%$ \\
\hline
\end{tabular}

\section{Conclusion}

In this paper, we improve Haar features and AdaBoost algorithm. Take advantage of skin color detection to settle the face region, and the new Haar features to improve the accuracy. Aiming at the traditional AdaBoost algorithm's excessive distribution, we introduce new the method of weight updating, which improves training efficiency, reduce the error detection rate. The experiment results show that this method has a good effect on face detection.

\section{References}

[1] E. Hjelmas and B. K. Low, Face Detection: A Survey, Computer Vision and Image Understanding, vol. 83, pp. 236-274, 2001.

[2] Y. Ming-Hsuan, D. J. Kriegman, and N. Ahuja, Detecting faces in images: a survey, Pattern Analysis and Machine Intelligence, IEEE Transactions on, vol. 24, pp. 34-58, 2002.

[3] Wang J G, Tam T N. A new face detection met based on shape information [J]. Pattern Recognition

[4] Yang G, Huang T S. Human face detection in a complex background[J]. Pattern recognition, 1994, 27(1): 53-63.

[5] Tofighi A, Monadjemi S A. Face detection and recognition using skin color and AdaBoost algorithm combined with Gabor features and SVM classifier[C]//Multimedia and Signal Processing (CMSP), 2011 International Conference on. IEEE, 2011, 1: 141-145.

[6] Kim J, Moon Y, Kim J, et al. Face detection method based on skin color and pattern match: U.S. Patent Application 11/195,611[P]. 2005-8-3.

[7] Freund Y, Schap Ire R. A Short Introduction to Boosting[J]. Journal of Japanese Society for Artificial Intelligence,1999,14 (5):771-780.

[8] Drucker H, Schapire R, Simard P. Boosting performance in neural networks[J].International Journal of Pattern Recognition Artificial Intel(1993,7(4):705 - 719.

[9] Lee J S, Kuo Y M, Chung P C, et al. Naked image detection based on adaptive and extensible skin color model[J]. Pattern recognition, 2007, 40(8): 2261-2270.

[10]P. Viola and M. Jones, Rapid object detection using a boosted cascade of simple features, in 
Computer Vision and Pattern Recognition, 2001. CVPR 2001. Proceedings of the 2001 IEEE Computer Society Conference on, 2001, pp. I-511-I-518 vol.1.

[11]Lienhart R, Maydt J. An Extended Set of Haar Features for Rapid Object Detection Proc of the IEEE International Conference on Image Processing. New York， USA， 2002， I: 900 $-903$

[12]Seul M, O'Gorman L, Sammon M J. Practical algorithms for image analysis: Descriptions, Examples, and Code[M]. London: Cambridge University Press,2008. 